\title{
TABLES DEDUCED FROM THE NEW EXPERIENCE TABLE $\mathrm{H}^{\mathrm{MF}}$ (ADJUSTED).
}

To the Eclitor of the Journal of the Institute of Actuaries.

Sir,-Having had, by the kind assistance of Mr. James Stark, junr., some monetary tables computed from the Table $\mathrm{H}^{\mathrm{MF}}$ of the "Mortality Experience of Life Assurance Companies collected by the Institute of Actuaries," I beg to place them at your disposal for publication in the Jurrnal.

They are calculated at 3 and 4 per cent. from the table of mortality adjusted by Mr. Woolhouse on Makeham's modification of Gompertz's theory, the formation of which table he has fully explained in the Journal, vol. Xv pp. 404-408; but I have used the logarithms throughout to five places only, considering them amply sufficient for ordinary official purnoses.

Mr. Makeham's formula expressing the law of mortality is

$$
\log l_{x}=\log k-x \log a+q^{x} \log g
$$

and the constants deduced from the original observations in Table $\mathrm{H}^{\mathrm{MFF}}$ are

$$
\begin{aligned}
& \log k=5 \cdot 04119 \\
& \log a=\cdot 00286 \\
& \log g=\cdot 00041 \\
& \log q=\cdot 04
\end{aligned}
$$

\begin{tabular}{|c|c|c|c|c|c|}
\hline \multirow[b]{2}{*}{ Age. } & \multicolumn{2}{|c|}{$\begin{array}{l}\text { TABLE HMF } \\
\text { NOUBER LIVING. }\end{array}$} & \multicolumn{3}{|c|}{$\begin{array}{l}\text { VALUE of Singlie LIFE ANNCtTy } \\
3 \text { PER CENT. }\end{array}$} \\
\hline & $\begin{array}{l}\text { Otiginal } \\
\text { Facts. }\end{array}$ & $\begin{array}{l}\text { Adjusted } \\
\text { Table. }\end{array}$ & $\begin{array}{c}\text { By New } \\
\text { Experience. }\end{array}$ & $\begin{array}{c}\text { By } \\
\text { Experience } \\
\text { of } 17 \text { Offices }\end{array}$ & $\begin{array}{l}\text { By Carlis'e } \\
\text { Table. }\end{array}$ \\
\hline 20 & 9554 & 9581 & $21 \cdot 956$ & $21 \cdot 797$ & $21 \cdot 694$ \\
\hline 30 & 8904 & 8890 & $19 \cdot 912$ & $19 \cdot 754$ & $10 \cdot 556$ \\
\hline 40 & 8128 & 8137 & $17 \cdot 254$ & $17 \cdot 123$ & $17 \cdot 143$ \\
\hline 50 & 7183 & 7198 & $14 \cdot 004$ & $13 \cdot 820$ & $14 \cdot 303$ \\
\hline 60 & 5847 & 5843 & $10 \cdot 368$ & $10 \cdot 188$ & $10 \cdot 491$ \\
\hline 70 & 3805 & 3822 & $6 \cdot 784$ & $6 \cdot 685$ & $7 \cdot 123$ \\
\hline 80 & 1411 & 1454 & $3 \cdot 789$ & 37799 & $4 \cdot 365$ \\
\hline 90 & 159 & 142 & $1 \cdot 729$ & $1 \cdot 516$ & $2 \cdot 499$ \\
\hline
\end{tabular}

The following will show the close approximation to the original facts of the number living at the decennial ages by the adjusted table, and also a comparison of the values of life annuities by the New Experience, by the Experience of 17 Offices, and by the Carlisle Table.

I remain, Sir, Yours obediently,

\section{Quardian Assurance Office,} SAMUEL BROWN. 11 Lombard Street, 31st Aug. 1871. 
TABLE OF MoRTality $\mathrm{H}^{\mathrm{MF}}$ (adjusted), showing the Number Living, the Number Dying, and the chance of dying in a Year, for every Age from 10 to 100 .

\begin{tabular}{|c|c|c|c|c|c|c|c|}
\hline$\underset{x}{A g e}$ & $l_{x}$ & $d_{x}$ & $1-p_{x}$ & $\underset{x}{\operatorname{Age}}$ & $l_{x}$ & $d_{x}$ & $1-p_{x}$ \\
\hline 10 & 102698 & 698 & .00679 & 56 & 64534 & 1430 & .02216 \\
\hline 11 & 102000 & 695 & .00682 & 57 & 63104 & 1493 & $\cdot 02366$ \\
\hline 12 & 101305 & 693 & $\cdot 00684$ & 58 & 61611 & 1557 & .02528 \\
\hline 13 & 100612 & 690 & $\cdot 00686$ & 59 & 60054 & 1627 & .02710 \\
\hline 14 & 99922 & 688 & .00688 & 60 & 58427 & 1696 & .02902 \\
\hline 15 & 99234 & 688 & .00693 & 61 & 56731 & 1769 & .03119 \\
\hline 16 & 98546 & 685 & .00695 & 62 & 54962 & 1842 & .03350 \\
\hline 17 & 97861 & 685 & .00700 & 63 & 53120 & 1916 & .03608 \\
\hline 18 & 97176 & 684 & .00704 & 64 & 51204 & 1992 & .03890 \\
\hline 19 & 96492 & 684 & .00709 & 65 & 49212 & 2063 & .01192 \\
\hline 20 & 95808 & 684 & .00714 & 66 & $47[49$ & 2136 & .04529 \\
\hline 21 & 95124 & 683 & .00718 & 67 & 45013 & 2203 & .04894 \\
\hline 22 & 94441 & 685 & .00725 & 68 & 42810 & 2266 & .05293 \\
\hline 23 & 93756 & 686 & .00732 & 69 & 40544 & 2322 & .05729 \\
\hline 24 & 93070 & 687 & .00739 & 70 & 38222 & 2372 & .06205 \\
\hline 25 & 92383 & 691 & .00748 & 71 & 35850 & 2410 & .06722 \\
\hline 26 & 91692 & 692 & .00755 & 72 & 33440 & 2437 & .07289 \\
\hline 27 & 91000 & 697 & .00766 & 73 & 31003 & 2451 & .07904 \\
\hline 28 & 90303 & 701 & .00775 & 74 & 28552 & 2448 & .08576 \\
\hline 29 & 89602 & 704 & .00787 & 75 & 26104 & 2429 & .09304 \\
\hline 30 & 88898 & 712 & .00800 & 76 & 23675 & 2391 & $\cdot 10098$ \\
\hline 31 & 88186 & 716 & $\cdot 00812$ & 77 & 21284 & 2333 & $\cdot 10961$ \\
\hline 32 & 87470 & 726 & .00830 & 78 & $1895 \mathrm{I}$ & 2254 & $\cdot 11897$ \\
\hline 33 & 86744 & 732 & .00844 & 79 & 16697 & 2156 & $\cdot 12910$ \\
\hline 34 & 86012 & 741 & .00862 & 80 & 14541 & 2037 & $\cdot 14010$ \\
\hline 35 & 85271 & 753 & -00883 & 81 & 12504 & 1901 & $\cdot 15199$ \\
\hline 36 & 84518 & 765 & $\cdot 00905$ & 82 & 10603 & 1747 & $\cdot 16484$ \\
\hline 37 & 83753 & 779 & $\cdot 00930$ & 83 & 8856 & 1583 & $\cdot 17872$ \\
\hline 38 & 82974 & 793 & $\cdot 00956$ & 84 & 7273 & 1408 & $\cdot 19364$ \\
\hline 39 & 82181 & 808 & .00983 & 85 & 5865 & 1230 & $\cdot 20972$ \\
\hline 40 & 81373 & 828 & .01017 & 86 & 4635 & 1053 & .22703 \\
\hline 41 & 80545 & 845 & .01049 & 87 & 3582 & 879 & .24541 \\
\hline 42 & 79700 & 868 & .01090 & 88 & 2703 & 717 & $\cdot 26522$ \\
\hline 43 & 78832 & 890 & .01129 & 89 & 1986 & 568 & .28628 \\
\hline 44 & 77942 & 917 & .01177 & 90 & 1418 & 438 & .30871 \\
\hline 45 & 77025 & 943 & .01224 & 91 & 980 & 326 & .33247 \\
\hline 46 & 76082 & 975 & .01281 & 92 & 654 & 234 & .35758 \\
\hline 47 & 75107 & 1007 & $\cdot 01340$ & 93 & 420 & 161 & .38406 \\
\hline 48 & 74100 & 1042 & .01406 & 94 & 259 & 107 & $\cdot 41181$ \\
\hline 49 & 73058 & 1080 & .01479 & 95 & 152 & 67 & $\cdot 44081$ \\
\hline 50 & 71978 & 1121 & 601558 & 96 & 85 & 40 & .47097 \\
\hline 51 & 70857 & 1164 & .01642 & 97 & 45 & 23 & .50217 \\
\hline 52 & 69693 & 1212 & .01739 & 98 & 22 & 12 & .53427 \\
\hline 53 & 68481 & 1261 & .01841 & 99 & 10 & 5 & .56714 \\
\hline 54 & 67220 & 1315 & $\cdot 0[958$ & 100 & 5 & 5 & 1 . \\
\hline 55 & 65905 & 1371 & $\cdot 02080$ & & & & \\
\hline
\end{tabular}


TABLE $\mathrm{H}^{\mathrm{MF}}$ (adjusted). Commutation Table, showing also the Value of the Life Annuity $a_{x}$ with Interest at 3 per-cent, for every Year of Age from 10 to 100.

\begin{tabular}{|c|c|c|c|c|}
\hline$\underset{x}{\text { Age }}$ & $\mathrm{D}_{x}$ & $\mathrm{~N}_{x}$ & $\mathrm{M}_{x}$ & $a_{x}$ \\
\hline 10 & $7647^{\circ}$ & $1793457^{\circ}$ & 21952 & $23 \cdot 469$ \\
\hline 11 & 73687. & $1719770^{\circ}$ & 21448 & 23.339 \\
\hline 12 & 71053. & $1648717^{\circ}$ & 20961 . & 23.204 \\
\hline 13 & $68513^{\circ}$ & $1580204^{\circ}$ & $20489^{\circ}$ & $23 \cdot 064$ \\
\hline 14 & $66060^{\circ}$ & $1514144^{\circ}$ & 20033 & $22 \cdot 921$ \\
\hline 15 & $63694^{\circ}$ & $1450450^{\circ}$ & $19591^{\circ}$ & 22.772 \\
\hline 16 & $61410^{\circ}$ & $1389040^{\circ}$ & 19162 & 22.619 \\
\hline 17 & 59208 . & 1329832 & $18748^{\circ}$ & $22 \cdot 161$ \\
\hline 18 & $57081^{\circ}$ & $1272751^{\circ}$ & $18346^{\circ}$ & $22 \cdot 297$ \\
\hline 19 & $55028^{\circ}$ & 1217723 & $17956^{\circ}$ & $22 \cdot 129$ \\
\hline 20 & $53047^{\circ}$ & $1164676^{\circ}$ & $17577^{\circ}$ & 21.956 \\
\hline 21 & $51134^{\circ}$ & 1113542 . & $17200^{\circ}$ & 21.777 \\
\hline 22 & $49288^{\circ}$ & 1064254 & 16853 & 21593 \\
\hline 23 & $47505^{\circ}$ & $1016749^{\circ}$ & $16506^{\circ}$ & $21 \cdot 403$ \\
\hline 24 & $45785^{\circ}$ & 970964 & $16169^{\circ}$ & $2 \mathrm{I} \cdot 208$ \\
\hline 25 & 44122 & 926842 & $15841^{\circ}$ & $2 l \cdot 006$ \\
\hline 26 & 42517 & $884325^{\circ}$ & $\mathrm{I} 552 \mathrm{I}^{\circ}$ & 20.799 \\
\hline 27 & 40967 & 843358 & 15209. & 20.586 \\
\hline 28 & $39469^{\circ}$ & $803889^{\circ}$ & $14904^{\circ}$ & 20.368 \\
\hline 29 & 38022 & $765867^{\circ}$ & $14607^{\circ}$ & $20 \cdot 142$ \\
\hline 30 & 36624. & 729243 & 14317. & $19 \cdot 611$ \\
\hline 31 & $35274^{\circ}$ & $693969^{\circ}$ & $14032^{\circ}$ & 99.674 \\
\hline 32 & 33968 . & 660001 . & 13754 . & $19 \cdot 430$ \\
\hline 33 & 32705. & $627296^{\circ}$ & $13480^{\circ}$ & $19 \cdot 181$ \\
\hline 34 & $31484^{\circ}$ & 595812 & $13212^{\circ}$ & 18924 \\
\hline 35 & 30304 & 565508 & $12949^{\circ}$ & $18 \cdot 661$ \\
\hline 36 & 29162 . & $536346^{\circ}$ & 12689. & ] $8 \cdot 392$ \\
\hline 37 & $28056^{\circ}$ & $508290^{\circ}$ & $12433^{\circ}$ & $18 \cdot 118$ \\
\hline 38 & $26986^{\circ}$ & $481304^{\circ}$ & $12180^{\circ}$ & 17.836 \\
\hline 39 & $25949^{\circ}$ & 455355 & $11930^{\circ}$ & $17 \cdot 548$ \\
\hline 40 & $24945^{\circ}$ & $430410^{\circ}$ & $11682^{\circ}$ & $17 \cdot 254$ \\
\hline 41 & $23972^{\circ}$ & 406438 & $11436^{\circ}$ & 16.954 \\
\hline 42 & $23030^{\circ}$ & $383408^{\circ}$ & $11] 92$. & 16.618 \\
\hline 43 & $22116^{\circ}$ & 361292 & $10948^{\circ}$ & 16337 \\
\hline 44 & $21229^{\circ}$ & 340063 & $10706^{\circ}$ & 16.019 \\
\hline 45 & 20368 . & 319695 & $10464 \cdot 0$ & 15696 \\
\hline 46 & 19533. & 300162 & $\mathrm{~J} 0221 \cdot 9$ & $15 \cdot 367$ \\
\hline 47 & $18721^{\circ}$ & 281441 . & 9978.9 & 15.034 \\
\hline 48 & $17932^{\circ}$ & 263509 . & $9735 \cdot 2$ & $14 \cdot 695$ \\
\hline 49 & $17165^{\circ}$ & $246344^{\circ}$ & $9 \pm 90.4$ & $14 \cdot 351$ \\
\hline 50 & $16419^{\circ}$ & 229925 . & 92440 & 14004 \\
\hline 51 & 15692 . & 214233 & $8995 \cdot 7$ & $\begin{array}{l}13.652 \\
132\end{array}$ \\
\hline 52 & $14985^{\circ}$ & $199248^{\circ}$ & 8745.4 & $13 \cdot 297$ \\
\hline 53 & $14296^{\circ}$ & 184952 & 8492.4 & $\begin{array}{l}10.938 \\
12.938\end{array}$ \\
\hline 54 & $13624^{\circ}$ & 171328. & 8236.8 & 12.576 \\
\hline 55 & 12968 . & $158360^{\circ}$ & 7978.0 & $12 \cdot 212$ \\
\hline 56 & 12328 . & 146032 . & $7716 \cdot 1$ & $11 \cdot 845$ \\
\hline 57 & 11704 . & 134328 . & 74509 & 11.474 \\
\hline 58 & $11094^{\circ}$ & $123234^{\circ}$ & $7182 \cdot 1$ & $11 \cdot 108$ \\
\hline 59 & $10+99^{\circ}$ & $132735^{\circ}$ & $6909 \cdot 9$ & 10738 \\
\hline 60 & 99170 & 102818 . & $6633 \cdot 7$ & $10 \cdot 368$ \\
\hline 61 & $9348 \cdot 7$ & $93469^{\circ}$ & 63542 & $9 \cdot 998$ \\
\hline 62 & $8793 \cdot 3$ & $84676^{\circ}$. & $6071 \cdot 2$ & 9630 \\
\hline 63 & $8251 \cdot 1$ & 76425 & $5785 \cdot 1$ & 9263 \\
\hline 64 & $7721 \cdot 8$ & 68703 . & $5496 \cdot 1$ & 8.897 \\
\hline 65 & $7205 \cdot 3$ & 61498 . & $5204 \cdot 4$ & 8535 \\
\hline 66 & $6702 \cdot 1$ & $54796^{\circ}$ & $4911 \cdot 1$ & $8 \cdot 176$ \\
\hline 67 & $6212 \cdot 3$ & $48584^{\circ}$ & $4616 \cdot 3$ & $7 \cdot 821$ \\
\hline
\end{tabular}


1872.] Tables deduced from the New Experience.

3 per-cent Commutation and Annuity Tables-(continued).

\begin{tabular}{|c|c|c|c|c|}
\hline$\underset{x}{\operatorname{Age}}$ & $D_{x}$ & $N_{x}$ & $\mathrm{M}_{x}$ & $a_{x}$ \\
\hline 68 & $5736 \cdot 1$ & $42848^{\circ}$ & $4321 \cdot 1$ & $7 \cdot 470$ \\
\hline 69 & $5274 \cdot 2$ & $37574^{\circ}$ & $4026 \cdot 3$ & $7 \cdot 124$ \\
\hline 70 & $4827 \cdot 3$ & $32747^{\circ}$ & $3733 \cdot 0$ & $6 \cdot 784$ \\
\hline 71 & $4395 \cdot 9$ & $28351^{\circ}$ & $3442 \cdot 1$ & $6 \cdot 449$ \\
\hline 72 & $3981 \cdot 0$ & $24370^{\circ}$ & $3155 \cdot 2$ & 6.122 \\
\hline 73 & $3583 \cdot 3$ & $20787^{\circ}$ & $2873 \cdot 5$ & $5 \cdot 801$ \\
\hline 74 & $3204 \cdot 0$ & 17583. & $2598 \cdot 5$ & $5 \cdot 488$ \\
\hline 75 & $2843 \cdot 9$ & 14739 . & $2331 \cdot 8$ & $5 \cdot 183$ \\
\hline 76 & $2504 \cdot 1$ & $12235^{\circ}$ & $2074 \cdot 9$ & $4 \cdot 886$ \\
\hline 77 & $2185 \cdot 7$ & $10049^{\circ}$ & $1829 \cdot 4$ & $4 \cdot 598$ \\
\hline 78 & $1889 \cdot 5$ & 81599 & 1596.8 & $4 \cdot 319$ \\
\hline 79 & $1616 \cdot 2$ & $6543 \cdot 7$ & $1378 \cdot 6$ & $4^{\circ} 049$ \\
\hline 80 & 1366.5 & $5177 \cdot 2$ & $1176^{\circ} 0$ & $3 \cdot 789$ \\
\hline 81 & $1140^{\prime} 9$ & $4036 \cdot 3$ & $990 \cdot 10$ & 3.538 \\
\hline 82 & $939 \cdot 29$ & 30970 & $82 I \cdot 70$ & 3.297 \\
\hline 83 & $761 \cdot 61$ & 2335.4 & $671 \cdot 45$ & $3 \cdot 066$ \\
\hline 84 & $607 \cdot 27$ & $1728 \cdot 1$ & $539 \cdot 27$ & $2 \cdot 846$ \\
\hline 85 & $475 \cdot 42$ & $1252 \cdot 7$ & $425 \cdot 13$ & $2 \cdot 635$ \\
\hline 86 & $364 \cdot 77$ & 887.89 & $328 \cdot 32$ & $2 \cdot 434$ \\
\hline 87 & $273 \cdot 74$ & $614 \cdot 15$ & $247 \cdot 86$ & $2 \cdot 244$ \\
\hline 88 & $200 \cdot 54$ & $413 \cdot 61$ & $182 \cdot 65$ & 2.062 \\
\hline 89 & $143 \cdot 07$ & 270.54 & $131 \cdot 01$ & 1.891 \\
\hline 90 & $99 \cdot 136$ & $171 \cdot 40$ & $91 \cdot 29$ & $1 \cdot 729$ \\
\hline 91 & 66.535 & $104 \cdot 86$ & $61 \cdot 55$ & 1.576 \\
\hline 92 & $43 \cdot 121$ & $61 \cdot 7 \nless 3$ & $40 \cdot 06$ & $I \cdot 432$ \\
\hline 93 & 26.895 & 34848 & $25-078$ & $I \cdot 296$ \\
\hline 94 & $16 \cdot 083$ & $18 \cdot 765$ & $15 \cdot 078$ & $1 \cdot 167$ \\
\hline 95 & $9 \cdot 1846$ & $9 \cdot 5803$ & $8 \cdot 624$ & $1 \cdot 043$ \\
\hline 96 & $4 \cdot 9864$ & $4 \cdot 5939$ & 4700 & $\cdot 921$ \\
\hline 97 & 2.5611 & $2 \cdot 0328$ & $2 \cdot 426$ & $\cdot 794$ \\
\hline 98 & $1 \cdot 2379$ & $\cdot 7949$ & $\cdot 1 \cdot 156$ & -642 \\
\hline 99 & 5597 & $\cdot 2352$ & $\cdot 513$ & $\cdot 420$ \\
\hline 100 & -2352 & $\cdots$ & -253 & $\cdot 000$ \\
\hline
\end{tabular}

TABLE $\mathrm{H}^{\mathrm{MF}}$ (adjusted). Values of Annuities on Two Joint Lives of Equal Ages, at 3 and at 4 per-cent Interest.

\begin{tabular}{|c|c|c|c|c|c|c|c|}
\hline \multicolumn{2}{|c|}{ Ages. } & $\begin{array}{c}\text { At } \\
\text { 3 per-cent } \\
\text { Interest. }\end{array}$ & $\begin{array}{c}\text { At } \\
\text { 4 per-cent } \\
\text { Interest. }\end{array}$ & \multicolumn{2}{|c|}{ Ages. } & $\begin{array}{l}\text { At } \\
\text { 3 per-cent } \\
\text { Interest. }\end{array}$ & $\begin{array}{l}\text { At } \\
\text { 4 per-cent } \\
\text { Interest. }\end{array}$ \\
\hline 10 & & $19 \cdot 861$ & 16.924 & 55 & 55 & $8 \cdot 971$ & $8 \cdot 349$ \\
\hline 11 & 11 & 19738 & 16843 & 56 & 56 & 8.637 & $8 \cdot 056$ \\
\hline 12 & 12 & $19 \cdot 610$ & 16.758 & 57 & 57 & 8:304 & $7 \cdot 763$ \\
\hline 13 & 13 & $19 \cdot 477$ & 16.669 & 58 & 58 & $7 \cdot 972$ & $7 \cdot 469$ \\
\hline 14 & 14 & $19 \cdot 340$ & 16.576 & 59 & 59 & $7 \cdot 643$ & $7 \cdot 176$ \\
\hline 15 & 15 & $19 \cdot 153$ & 16.479 & 60 & 60 & 7317 & 6885 \\
\hline 16 & 16 & $19 \cdot 050$ & 16.378 & 61 & 61 & 6.994 & 6.595 \\
\hline 17 & 17 & 18.897 & 16.273 & 62 & 62 & 6.675 & $6: 307$ \\
\hline 18 & 18 & 18.739 & $16 \cdot 163$ & 63 & 63 & $6 \cdot 360$ & 6.021 \\
\hline 19 & 19 & $18 \cdot 576$ & 16049 & 64 & 64 & 6.050 & $5 \cdot 740$ \\
\hline 20 & 20 & $18 \cdot 408$ & 15.930 & 65 & 65 & $5 \cdot 746$ & $5 \cdot 463$ \\
\hline 21 & 21 & $18 \cdot 233$ & $15 \cdot 806$ & 66 & 66 & 5.448 & $5 \cdot 189$ \\
\hline 22 & 22 & $18 \cdot 053$ & $15 \cdot 677$ & 67 & 67 & $5 \cdot 156$ & 4921 \\
\hline 23 & 23 & $17 \cdot 867$ & $15 \cdot 544$ & 68 & 68 & 4872 & 4.658 \\
\hline 24 & 24 & $17 \cdot 675$ & $15 \cdot 404$ & 69 & 69 & $4 \cdot 594$ & $4.40 \mathrm{l}$ \\
\hline 25 & 25 & $17 \cdot 478$ & $15 \cdot 260$ & 70 & 70 & 4.325 & $4 \cdot 150$ \\
\hline 26 & 26 & $17-275$ & $15 \cdot 111$ & 71 & 71 & 4.063 & $\mathbf{3} 906$ \\
\hline 27 & 27 & 17.065 & $14 \cdot 954$ & 72 & 72 & 38810 & 3669 \\
\hline 28 & 28. & $16 \cdot 849$ & $14 \cdot 794$ & 73 & 73 & $3 \cdot 566$ & $3 \cdot 440$ \\
\hline
\end{tabular}


Joint Life Annuities, 3 and 4 per-cent-(continued).

\begin{tabular}{|c|c|c|c|c|c|c|c|}
\hline \multicolumn{2}{|c|}{ Ages. } & \multirow{2}{*}{$\frac{\begin{array}{c}\text { At } \\
\text { 3 per-cent } \\
\text { Interest. }\end{array}}{16.626}$} & \multirow{2}{*}{$\frac{\begin{array}{c}\text { At } \\
4 \text { per-cent } \\
\text { Interest. }\end{array}}{14 \cdot 627}$} & \multicolumn{2}{|c|}{ Ages. } & \multirow{2}{*}{$\begin{array}{c}\begin{array}{c}\text { At } \\
\text { 3 per-cent } \\
\text { Interest. }\end{array} \\
3 \cdot 330\end{array}$} & \multirow{2}{*}{$\begin{array}{c}\begin{array}{c}\text { At } \\
\text { 4 per-cent } \\
\text { Interest. }\end{array} \\
3 \cdot 218\end{array}$} \\
\hline 29 & 29 & & & 74 & 74 & & \\
\hline 30 & 30 & $16^{*} 398$ & $14: 454$ & 75 & 75 & $3 \cdot 104$ & $3 \cdot 003$ \\
\hline 31 & 31 & $16 \cdot 163$ & 14276 & 76 & 76 & $2 \cdot 886$ & $2 \cdot 797$ \\
\hline 32 & 32 & $15 \cdot 922$ & 14.091 & 77 & 77 & 2678 & $2 \cdot 599$ \\
\hline 33 & 33 & $15 \cdot 675$ & $13 \cdot 901$ & 78 & 78 & $2 \cdot 479$ & $2 \cdot 410$ \\
\hline 34 & 34 & $15 \cdot 422$ & 13.704 & 79 & 79 & $2 \cdot 290$ & 2229 \\
\hline 35 & 35 & 15162 & 13.501 & 80 & 80 & $2 \cdot 110$ & $2 \cdot 056$ \\
\hline 36 & 36 & $14 \cdot 896$ & $13 \cdot 292$ & 81 & 81 & 1.939 & $1 \cdot 892$ \\
\hline 37 & 37 & $14 \cdot 624$ & 13078 & 82 & 82 & 1.777 & 1736 \\
\hline 38 & 38 & $14 \cdot 348$ & 12.858 & 83 & 83 & $1 \cdot 624$ & $1 \cdot 588$ \\
\hline 39 & 39 & 14.065 & $12 \cdot 631$ & 84 & 84 & 1.480 & $1 \cdot 449$ \\
\hline 40 & 40 & 13.776 & $12 \cdot 399$ & 85 & 85 & $1 \cdot 345$ & $1 \cdot 318$ \\
\hline 41 & 41 & $13 \cdot 482$ & $12 \cdot 161$ & 86 & 86 & 1218 & $1 \cdot 194$ \\
\hline 42 & 42 & $13 \cdot 182$ & 11.917 & 87 & 87 & $1 \cdot 099$ & $1 \cdot 079$ \\
\hline 43 & 43 & 12.879 & $11 \cdot 668$ & 88 & 88 & .988 & .971 \\
\hline 44 & 44 & $12 \cdot 570$ & $11 \cdot 414$ & 89 & 89 & $\cdot 885$ & 870 \\
\hline 45 & 45 & $12 \cdot 257$ & $11 \cdot 154$ & 90 & 90 & .789 & $\cdot 776$ \\
\hline 46 & 46 & $11 \cdot 940$ & $10 \cdot 890$ & 91 & 91 & .700 & .690 \\
\hline 47 & 47 & $11 \cdot 619$ & $10 \cdot 622$ & 92 & 92 & .618 & $\cdot 609$ \\
\hline 48 & 48 & $11 \cdot 295$ & $10 \cdot 349$ & 93 & 93 & -543 & $\cdot 535$ \\
\hline 49 & 49 & $10 \cdot 968$ & 10.072 & 94 & 94 & 474 & $\cdot 468$ \\
\hline 50 & 50 & $10 \cdot 639$ & $9 \cdot 792$ & 95 & 95 & .411 & $\cdot 406$ \\
\hline 51 & 51 & $10 \cdot 308$ & $9 \cdot 508$ & 96 & 96 & $\cdot 354$ & $\cdot 349$ \\
\hline 52 & 52 & $9 \cdot 975$ & $9 \cdot 221$ & 97 & 97 & .301 & 297 \\
\hline 53 & 53 & $9 \cdot 640$ & 8.933 & 98 & 98 & .249 & 246 \\
\hline 54 & 54 & $9 \cdot 306$ & $8 \cdot 642$ & 99 & 99 & $\cdot 182$ & $\cdot 180$ \\
\hline
\end{tabular}

TABLE $\mathrm{H}^{\mathrm{MF}}$ (adjusted). Commutation Table, showing also the Value of the Life Annuity $a_{x}$ with Interest at 4 per-cent, for every Year of Age from 10 to 100 .

\begin{tabular}{|c|c|c|c|c|}
\hline$\underset{x}{\mathrm{Age}}$ & $\mathrm{D}_{x}$ & $\mathrm{~N}_{x}$ & $\mathrm{M}_{x}$ & $a_{x}$ \\
\hline 10 & 69379 . & $1354883^{\circ}$ & $14599 \cdot 9$ & $19 \cdot 529$ \\
\hline 11 & $66257^{\circ}$ & $1288626^{\circ}$ & $14146 \cdot 5$ & $19 \cdot 449$ \\
\hline 12 & $63275^{\circ}$ & 1225351 & $13712 \cdot 4$ & $19 \cdot 366$ \\
\hline 13 & $60425^{\circ}$ & $1164926^{\circ}$ & 13296.2 & $19 \cdot 279$ \\
\hline 14 & 57702 & $1107224^{\circ}$ & $12897 \cdot 7$ & $19 \cdot 189$ \\
\hline 15 & $55101^{\circ}$ & $1052123^{\circ}$ & 125157 & $19 \cdot 095$ \\
\hline 16 & 52615 & $999508^{\circ}$ & 12148.4 & 18.997 \\
\hline 17 & $50239^{\circ}$ & $949269^{\circ}$ & 117967 & $18 \cdot 895$ \\
\hline 18 & 47969. & $901300^{\circ}$ & 11458.6 & $18 \cdot 789$ \\
\hline 19 & $45799^{\circ}$ & 855501. & 111339 & 18.679 \\
\hline 20 & $43725^{\circ}$ & $811776^{\circ}$ & $10821 \cdot 7$ & $18: 566$ \\
\hline 21 & $41744^{\circ}$ & 770032 & $10521 \cdot 5$ & $18 \cdot 447$ \\
\hline 22 & $39850^{\circ}$ & 730182. & 102333 & $18 \cdot 323$ \\
\hline 23 & $38039^{\circ}$ & $692143^{\circ}$ & $9955 \cdot 4$ & $18 \cdot 196$ \\
\hline 24 & $36309^{\circ}$ & 655834 & $9687 \cdot 8$ & $18 \cdot 063$ \\
\hline 25 & $34655^{\circ}$ & $621179^{\circ}$ & $9430 \cdot 1$ & $17 \cdot 925$ \\
\hline 26 & 33072 & $588107^{\circ}$ & $9180 \cdot 9$ & $17 \cdot 783$ \\
\hline 27 & $31560^{\circ}$ & $556547^{\circ}$ & $8940 \cdot 9$ & $17 \cdot 634$ \\
\hline 28 & $30114^{\circ}$ & 526433 & $8708 \cdot 5$ & $17 \cdot 481$ \\
\hline 29 & $28731^{\circ}$ & 497702 & $8483 \cdot 7$ & $17 \cdot 323$ \\
\hline 30 & $27409^{\circ}$ & 470293 & $8266^{\circ} 6$ & $17 \cdot 159$ \\
\hline 31 & $26144^{\circ}$ & 444149 & $8055 \cdot 5$ & 16.989 \\
\hline 32 & $24934^{\circ}$ & $419215^{\circ}$ & $7851 \cdot 4$ & 16.813 \\
\hline 33 & $23776^{\circ}$ & $395439^{\circ}$ & $7652 \cdot 4$ & 16.632 \\
\hline 34 & $22669^{*}$ & $372770^{\circ}$ & $7459 \cdot 5$ & $16 \cdot 444$ \\
\hline 35 & $21609^{\circ}$ & $351161^{\circ}$ & $7271 \cdot 7$ & $16 \cdot 251$ \\
\hline 36 & $20594^{\circ}$ & $330567^{\circ}$ & $7088 \cdot 2$ & 16.051 \\
\hline 37 & 19623. & $310944^{\circ}$ & $6909 \cdot 0$ & $15 \cdot 846$ \\
\hline
\end{tabular}


1872.] Tables deduced from the New Experience.

4 per-cent Commutation and Annuity Tables-(continued).

\begin{tabular}{|c|c|c|c|c|}
\hline Age & $\mathbf{D}_{x}$ & $\mathbf{N}_{x}$ & $\mathbf{M}_{x}$ & $a_{x}$ \\
\hline 38 & 18693 & $292251^{\circ}$ & $6733 \cdot 5$ & 15.635 \\
\hline 39 & 17802. & $274449^{\circ}$ & $6561 \cdot 7$ & $15 \cdot 417$ \\
\hline 40 & $16949^{\circ}$ & $257500^{\circ}$ & $6393 \cdot 4$ & $15 \cdot 193$ \\
\hline 41 & $16131^{\circ}$ & $241369^{\circ}$ & $6227 \cdot 6$ & $14 \cdot 963$ \\
\hline 42 & $15348^{\circ}$ & $226021^{\circ}$ & $6064 \cdot 9$ & 14726 \\
\hline 43 & $14597^{\circ}$ & $211424^{\circ}$ & $5904 \cdot 2$ & $14 \cdot 484$ \\
\hline 44 & $13877^{\circ}$ & $197547^{\circ}$ & $5745^{\circ} 7$ & 14235 \\
\hline 45 & $13187^{\circ}$ & $184360^{\circ}$ & $5588 \cdot 7$ & $13 \cdot 981$ \\
\hline 46 & $12524^{\circ}$ & $171836^{\circ}$ & $5433 \cdot 5$ & $13 \cdot 720$ \\
\hline 47 & $11888^{\circ}$ & 159948 & 52792 & 13.455 \\
\hline 48 & $11278^{\circ}$ & $148670^{\circ}$ & $5125 \cdot 9$ & $13 \cdot 183$ \\
\hline 49 & 10692 & $137978^{\circ}$ & $4973 \cdot 4$ & $12 \cdot 905$ \\
\hline 50 & $10128^{\circ}$ & $127850^{\circ}$ & $4821 \cdot 4$ & $12 \cdot 623$ \\
\hline 51 & 9586.9 & $118263^{\circ}$ & $4669 \cdot 7$ & $12 \cdot 336$ \\
\hline 52 & $9066^{\circ} 9$ & $109196^{\circ}$ & $4518 \cdot 3$ & $12 \cdot 043$ \\
\hline 53 & $8566^{\circ} 4$ & $100630^{\circ}$ & $4366 \cdot 7$ & $11 \cdot 747$ \\
\hline 54 & $8085 \cdot 4$ & 925442 & $4215 \cdot 0$ & $11 \cdot 446$ \\
\hline 55 & $7622 \cdot 4$ & $84921 \cdot 8$ & $4062 \cdot 9$ & $11 \cdot 141$ \\
\hline 56 & $7176 \cdot 6$ & 77745.2 & $3910 \cdot 4$ & 10.833 \\
\hline 57 & $6747 \cdot 8$ & $70997 \cdot 4$ & $3757 \cdot 5$ & 10.522 \\
\hline 58 & $6334 \cdot 8$ & $64662 \cdot 6$ & $3604 \cdot 0$ & ] 0.208 \\
\hline 59 & 5937.0 & $58725 \cdot 6$ & $3450 \cdot 1$ & 9.891 \\
\hline 60 & $5554 \cdot 1$ & $53171 \cdot 5$ & $3295 \cdot 4$ & 9573 \\
\hline 61 & 5185.5 & $47986 \cdot 0$ & $3140 \cdot 4$ & $9 \cdot 254$ \\
\hline 62 & $4830 \cdot 5$ & 43155.5 & $2984 \cdot 9$ & $8 \cdot 934$ \\
\hline 63 & $4489 \cdot 1$ & $38666^{\circ} 4$ & $2829 \cdot 2$ & $8 \cdot 613$ \\
\hline 64 & 41607 & $34505 \cdot 7$ & $2673 \cdot 5$ & $8 \cdot 293$ \\
\hline 65 & 38450 & $30660 \cdot 7$ & $2517 \cdot 9$ & $7 \cdot 974$ \\
\hline 66 & $3542 \cdot 2$ & 27118.5 & $2362 \cdot 9$ & $7 \cdot 656$ \\
\hline 67 & $3251 \cdot 7$ & $23866^{\circ} 8$ & $2208 \cdot 6$ & $7 \cdot 340$ \\
\hline 68 & $2973 \cdot 6$ & $20893 \cdot 2$ & $2055 \cdot 6$ & $7 \cdot 026$ \\
\hline 69 & $2707 \cdot 9$ & 18185.3 & $1904 \cdot 3$ & 6.716 \\
\hline 70 & $2454 \cdot 6$ & $15730 \cdot 7$ & $1755 \cdot 2$ & $6 \cdot 409$ \\
\hline 71 & 221377 & $13517 \cdot 0$ & $1608 \cdot 7$ & $6 \cdot 106$ \\
\hline 72 & 1985.5 & 11531.5 & 14656 & $5 \cdot 808$ \\
\hline 73 & $1770 \cdot 0$ & $9761 \cdot 5$ & $1326 \cdot 5$ & $5 \cdot 515$ \\
\hline 74 & $1567 \cdot 4$ & $8194 \cdot 1$ & $1191 \cdot 9$ & $5 \cdot 228$ \\
\hline 75 & $1377 \cdot 8$ & 6816.3 & $1062 \cdot 7$ & $4 \cdot 947$ \\
\hline 76 & $1201 \cdot 6$ & $5614 \cdot 7$ & $939 \cdot 45$ & $4 \cdot 673$ \\
\hline 77 & $1038 \cdot 7$ & $4576^{\circ} 0$ & $822 \cdot 77$ & $4 \cdot 406$ \\
\hline 78 & $889 \cdot 28$ & $3686 \cdot 7$ & $713 \cdot 30$ & $4 \cdot 146$ \\
\hline 79 & $753 \cdot 36$ & $2933 \cdot 3$ & $611 \cdot 60$ & $3 \cdot 894$ \\
\hline 80 & $630 \cdot 86$ & $2302 \cdot 4$ & 518.06 & 3.650 \\
\hline 81 & $521 \cdot 62$ & $1780 \cdot 8$ & $433 \cdot 08$ & $3 \cdot 414$ \\
\hline 82 & $425 \cdot 32$ & $1355 \cdot 5$ & 356.83 & $3 \cdot 187$ \\
\hline 83 & $341 \cdot 55$ & 1013.9 & $289 \cdot 45$ & 2.969 \\
\hline 84 & $269 \cdot 72$ & $744 \cdot 16$ & $230 \cdot 74$ & $2 \cdot 759$ \\
\hline 85 & $209 \cdot 13$ & $535 \cdot 03$ & 180.53 & $2 \cdot 558$ \\
\hline 86 & $158 \cdot 91$ & $376 \cdot 12$ & $138 \cdot 36$ & $2 \cdot 367$ \\
\hline 87 & $118 \cdot 11$ & $258 \cdot 01$ & $103 \cdot 64$ & $2 \cdot 185$ \\
\hline 88 & $85 \cdot 696$ & $172 \cdot 31$ & $75 \cdot 77$ & 2.011 \\
\hline 89 & 60.545 & $111 \cdot 76$ & 53.91 & 1.846 \\
\hline 90 & $41 \cdot 551$ & $70 \cdot 210$ & $37 \cdot 26$ & 1.690 \\
\hline 91 & $27 \cdot 619$ & $42 \cdot 591$ & 24.920 & $1 \cdot 542$ \\
\hline 92 & $17 \cdot 727$ & $24 \cdot 864$ & 16.086 & $1 \cdot 403$ \\
\hline 93 & 10950 & 13.914 & $9 \cdot 989$ & $1 \cdot 271$ \\
\hline 94 & 6.4856 & $7 \cdot 4283$ & 5.955 & $1 \cdot 145$ \\
\hline 95 & $3 \cdot 6680$ & $3 \cdot 7603$ & $3 \cdot 377$ & 1.025 \\
\hline 96 & 1.9722 & $1 \cdot 7881$ & 1.825 & $\cdot 907$ \\
\hline 97 & $\mathrm{I} \cdot 0033$ & $\cdot 7848$ & .934 & .782 \\
\hline 98 & $\cdot 4802$ & $\cdot 3046$ & $\cdot 441$ & .634 \\
\hline 99 & 2151 & $\cdot 0895$ & $\cdot 194$ & $\cdot 416$ \\
\hline 100 & $\cdot 0895$ & $\ldots$ & $\cdot 095$ & \\
\hline
\end{tabular}

